\title{
Multilevel Assessment of Atherosclerotic Extent Using a 40-Section Multidetector Scanner after Transient Ischemic Attack or Ischemic Stroke
}

\author{
L. Mechtouff, L. Boussel, S. Cakmak, J.-L. Lamboley, M. Bourhis, N. Boublay, A.-M. Schott, L. Derex, T.-H. Cho,
}

N. Nighoghossian, and P.C. Douek

\begin{abstract}
BACKGROUND AND PURPOSE: The first part of this study assessed the potential of MDCT with a CTA examination of the aorta and the coronary, cervical, and intracranial vessels in the etiologic work-up of TIA or ischemic stroke compared with established imaging methods. The objective of the second part of this study was to assess the atherosclerotic extent by use of MDCT in these patients.
\end{abstract}

MATERIALS AND METHODS: From August 2007 to August 2011, a total of 96 patients with ischemic stroke or TIA without an evident cardioembolic source were enrolled. All patients underwent MDCT. Atherosclerotic extent was classified in 0, 1, 2, 3, and 4 atherosclerotic levels according to the number of arterial territories (aortic arch, coronary, cervical, intracranial) affected by atherosclerosis defined as $\geq 50 \%$ cervical, intracranial, or coronary stenosis or $\geq 4$-mm aortic arch plaque.

RESULTS: There were 91 patients who had an interpretable MDCT. Mean age was 67.4 years ( \pm 11 years), and 75 patients ( $83.3 \%$ ) were men. The prevalence of $0,1,2,3$, and 4 atherosclerotic levels was $48.3 \%, 35.2 \%, 12.1 \%, 4.4 \%$, and $0 \%$, respectively. Aortic arch atheroma was found in $47.6 \%$ of patients with 1 atherosclerotic level. The combination of aortic arch atheroma and cervical stenosis was found in $63.6 \%$ of patients with $\geq 2$ atherosclerotic levels. Patients with $\geq 2$ atherosclerotic levels were older than patients with $<2$ atherosclerotic levels $(P=.04)$ in univariate analysis.

CONCLUSIONS: MDCT might be useful to assess the extent of atherosclerosis. It could help to screen for high-risk patients who could benefit from a more aggressive preventive strategy.

ABBREVIATION: ECG = electrocardiogram

A ssessment of atherosclerosis from the heart to the brain in patients with stroke may allow an optimal selection of highrisk patients who could benefit from a more aggressive preventive strategy. Several studies have evaluated the accuracy of MDCT

Received February 21, 2013; accepted after revision April 25.

From the Stroke Unit (L.M., L.D., T.-H.C., N.N.), Hôpital Pierre Wertheimer, Hospices Civils de Lyon, Lyon, France; Radiology Department (L.B.), Hôpital de la CroixRousse, Hospices Civils de Lyon, Lyon, France; CNRS UMR 5220-INSERM U1044Université Lyon 1, Villeurbanne, France (L.B., L.D., T.-H.C., N.N., P.C.D.); Stroke Unit (S.C.), Centre Hospitalier Villefranche-sur-Saône, France; Radiology Department (J.-L.L.), Hôpital d'instruction des Armées Desgenettes, Lyon, France; Cellule Recherche PAM Imagerie (M.B.), Hospices Civils de Lyon, Lyon, France; Pôle Information Médicale Evaluation Recherche (N.B.), Hospices Civils de Lyon, Lyon, France; Equipe d'Accueil 4129, Université Lyon 1, Villeurbanne, France; Hospices Civils de Lyon (A.-M.S.), Pôle Information Médicale Evaluation Recherche, RECIF, Université Lyon 1, Villeurbanne, France.

This work was supported by the Hospices Civils de Lyon.

Abstract previously presented at: 17th French Neurovascular Society meeting, European Stroke Conference 2012.

Please send correspondence to Laura Mechtouff, Stroke Unit, Hôpital Pierre Wertheimer, Hospices Civils de Lyon, 59 Boulevard Pinel, 69677 Lyon Bron CEDEX France; e-mail: laura.mechtouff@chu-lyon.fr

http://dx.doi.org/10.3174/ajnr.A3760 with CTA examination of the aorta and coronary, cervical, and intracranial vessels to detect atherosclerosis. ${ }^{1-5}$

The first part of our study was a single-center, prospective, open-pilot study that was designed to assess MDCT with a CTA examination of the aorta and coronary, cervical, and intracranial vessels in the etiologic work-up of TIA and acute ischemic stroke compared with established imaging methods. Clinical and radiologic methods have been described elsewhere. ${ }^{6}$ In brief, patients 28-90 years old who were admitted to the hospital for a recent TIA or acute ischemic stroke without evident cardioembolic source in the Lyon Stroke Unit between August 1, 2007, and April 30,2008 , were included in this study. The period of inclusion was later extended to August 1, 2011. All patients had an MDCT examination with CTA of the aorta and coronary, cervical, and intracranial vessels compared with transthoracic echocardiography and transesophageal echocardiography, duplex ultrasonography of the cervical vessels, and MRA of the cervical and intracranial vessels. MDCT was not a part of the initial acute stroke assessment but was done within 7 days. We obtained approval from our local ethics committee and institutional review board and informed 
consent from each patient. It has been demonstrated that MDCT is feasible and accurate for the identification of stroke causes though its sensitivity for the detection of minor cardiac sources is limited.

The objective of the second part of our study was to assess the global atherosclerotic extent by using MDCT in these patients.

\section{MATERIALS AND METHODS Research Design}

In the second part of our study, we used data collected in the first part of the study. ${ }^{6}$

\section{Imaging Protocols}

We performed contrast-enhanced MDCT by using a Brilliance 40 scanner (Philips Healthcare, Best, the Netherlands), with iomeprol (Iomeron 400; Bracco Diagnostics, Milan, Italy) injected into the right cubital vein with an 18-gauge catheter. The patient was placed in the supine, head-first position. A 2-step protocol was performed: first, electrocardiogram (ECG)-gated aortic and heart acquisitions were performed in the head-to-feet direction, encompassing the aortic and heart area from the top of the aortic arch to the diaphragm. The following parameters were used: 40 detectors, individual detector width of $0.625 \mathrm{~mm}$, retrospective ECG gating, tube voltage of $120 \mathrm{kV}$, tube current of $300 \mathrm{mAs}$, pitch of 0.2 , and half-rotation reconstruction. Iomeprol $70 \mathrm{~mL}$ and then saline solution $60 \mathrm{~mL}$ were injected at $4 \mathrm{~mL} / \mathrm{s}$. A bolus-tracking method was used with an attenuation threshold of 200 Hounsfield units in the ascending aorta. Reconstruction parameters for the axial sections were a 1.5-mm effective section thickness, 1 - $\mathrm{mm}$ increments, a reconstruction filter Cardiac B, and an adapted field of view. Retrospective ECG-gated reconstruction was performed at $40 \%$ and $75 \%$ of the R-R interval. Then, 2 minutes later, a non-ECGgated acquisition from the aortic arch to the vertex (approximately $50 \mathrm{~cm}$ ) was performed with the following parameters: feet-to-head direction, section thickness of $1.2 \mathrm{~mm}$, pitch of 1.2 , tube voltage of $120 \mathrm{kV}$, amperage of $300 \mathrm{mAs}$ per section, reconstruction filter $\mathrm{B}$, and the bolus tracker set on the aortic arch with an attenuation threshold at 200 Hounsfield units. Iomeprol $50 \mathrm{~mL}$ and then saline solution $60 \mathrm{~mL}$ were injected at $4 \mathrm{~mL} / \mathrm{s}$, for a total injected contrast material volume of 120 $\mathrm{mL}$. The patient underwent imaging with the arms over the head during the aortic and heart acquisitions and with the arms at the sides during the second acquisition. General guidelines for ECG-gated cardiac MDCT were followed regarding the qualifications of the personnel, radiation dose monitoring, and the safety rules for contrast agent and $\beta$-blocker administration. $^{7-9} \beta$-Blockers (esmolol hydrochloride; Brevibloc; Baxter, Deerfield, Illinois) (0.5-1.0 mg per kilogram of body weight) were administered intravenously, if necessary, when the heart rate was higher than 80 beats per minute. CT was performed even in cases of atrial fibrillation. The total radiation dose and the heart rate of each patient during the examination were recorded. The thyroid gland was irradiated once during the acquisition from the aortic arch to the vertex.

\section{Imaging Evaluation}

The diagnostic work-up was focused on the following vascular abnormalities by use of MDCT with CTA:

- Atherosclerotic lesion of the carotid arteries leading to $\geq 50 \%$ stenosis. The degree of carotid stenosis was measured with the North American Symptomatic Carotid Endarterectomy Trial (NASCET) criteria. ${ }^{10}$ A measure (measure 1) was taken of the diameter of the narrowest portion of the cervical ICA in the axial plane. This was compared with the maximal diameter (measure 2) of the cervical ICA distal to the carotid bulb at a location in which the imaging plane was orthogonal to the artery, the arterial walls were parallel, and where there was no arterial disease. The degree of cross-sectional stenosis was calculated in percent as: percent stenosis $(1-$ [measure $1 /$ measure 2]) $\times 100 \%$, analogous to the method used in NASCET. In cases of poststenotic dilation of the ICA, we used as a denominator for the ratio calculation the diameter of the internal carotid well beyond the bulb, where the walls are parallel. Nearocclusion cases were defined as the presence of notable stenosis of the ICA bulb and distal ICA caliber reduction, compared with 1) expected size, 2) the controlateral ICA, and 3) the ipsilateral external carotid artery). In these cases, we did not do a ratio calculation and arbitrarily assigned as $95 \%$ stenosis as established in NASCET. ${ }^{10-12}$

- Atherosclerotic lesion of the vertebral arteries leading to $\geq 50 \%$ stenosis

- Aortic arch atheroma of $>4 \mathrm{~mm}$

- Intracranial artery stenosis $\geq 50 \%$

- Coronary artery stenosis $\geq 50 \%$

Atherosclerotic extent was classified in $0,1,2,3$, and 4 atherosclerotic levels according to the number of arterial territories (aortic, coronary, cervical, intracranial) affected by at least 1 vascular abnormality as we described previously.

\section{Statistical Analysis}

Continuous variables were expressed as mean (standard deviation), and categoric variables were expressed as percentages. We compared continuous variables by using the $t$ test or the MannWhitney test where appropriate, and categoric variables by using the Pearson $\chi^{2}$ test or the Fisher exact test where appropriate. The associations between atherosclerotic extent $(<2$ vs $\geq 2$ atherosclerotic levels) and main vascular risk factors were measured by calculation of adjusted odds ratios and 95\% confidence intervals by logistic regression analyses.

Multivariable models were adjusted for age, sex, diabetes, hypertension, dyslipidemia, and tobacco. The distribution of arterial disease combinations in patients with $\geq 2$ atherosclerotic levels was compared by use of the Fisher exact test. A $P$ value $<.05$ was considered statistically significant.

We performed statistical analysis by using STATA, version 11.0 (StataCorp, College Station, Texas) and R software, version 2.10.1 (http://www.r-project.org/).

\section{RESULTS}

Ninety-six patients were included. MDCT with CTA examination of the heart, aorta, and the cervical and intracranial vessels was not 
Table 1: Demographic and medical data according to number of atherosclerotic levels in 91 patients

\begin{tabular}{lccccc}
\hline $\begin{array}{c}\text { Number of atherosclerotic } \\
\text { levels }\end{array}$ & $\mathbf{0}$ & $\mathbf{1}$ & $\mathbf{2}$ & $\mathbf{3}$ & All \\
\hline Number of patients, $n(\%)$ & $44(48.3 \%)$ & $32(35.2 \%)$ & $11(12.1 \%)$ & $4(4.4 \%)$ & 91 \\
Age, $\mathrm{y}$, mean (SD) & $63.6(10.6)$ & $70.1(10.3)$ & $72.5(10.8)$ & $72.5(10.0)$ & $67.4(11.0)$ \\
Male sex, $n$ (\%) & $36(83.7)$ & $25(78.1)$ & $10(90.9))$ & $4(100.0)$ & $75(83.3)$ \\
TIA, $n$ (\%) & $27(62.8)$ & $17(53.1)$ & $6(54.6)$ & $3(75.0)$ & $53(58.9)$ \\
Stroke & & & & & \\
Initial NIHSS, mean (SD) & $5.2(3.9)$ & $7.7(5.4)$ & $6.5(5.8)$ & $0.3(0.6)$ & $6.1(4.9)$ \\
Hypertension, $n(\%)$ & $15(34.9)$ & $16(50.0)$ & $5(45.5)$ & $2(50.0)$ & $38(42.2)$ \\
LDL cholesterol, g/L & $1.23(0.3)$ & $1.20(0.4)$ & $1.14(0.3)$ & $1.50(0.4)$ & $1.22(0.4)$ \\
Smoking, $n(\%)$ & & & & & \\
$\quad$ Former & $7(16.3)$ & $8(25.8)$ & $3(27.3)$ & $1(25.0)$ & $19(21.4)$ \\
$\quad$ Current & $11(25.6)$ & $6(19.3)$ & $2(18.2)$ & $2(50.0)$ & $21(23.6)$ \\
$\quad$ Never & $25(58.1)$ & $17(54.8)$ & $6(54.6)$ & $1(25.0)$ & $49(55.1)$ \\
CRP, mg/L (\%) & $10.0(27.0)$ & $6.1(7.5)$ & $4.5(4.3)$ & $3.0(1.6)$ & $7.7(19.6)$ \\
Fibrinogen, g/L (\%) & $3.4(1.0)$ & $3.6(0.8)$ & $3.2(1.1)$ & $3.6(0.3)$ & $3.5(1.0)$ \\
History of vascular disease (\%) & $9(20.9)$ & $11(35.5)$ & $6(54.6)$ & 0 & $26(29.2)$ \\
\hline Nite
\end{tabular}

Note:-CRP indicates C-reactive protein; LDL, low-density lipoprotein; SD, standard deviation.
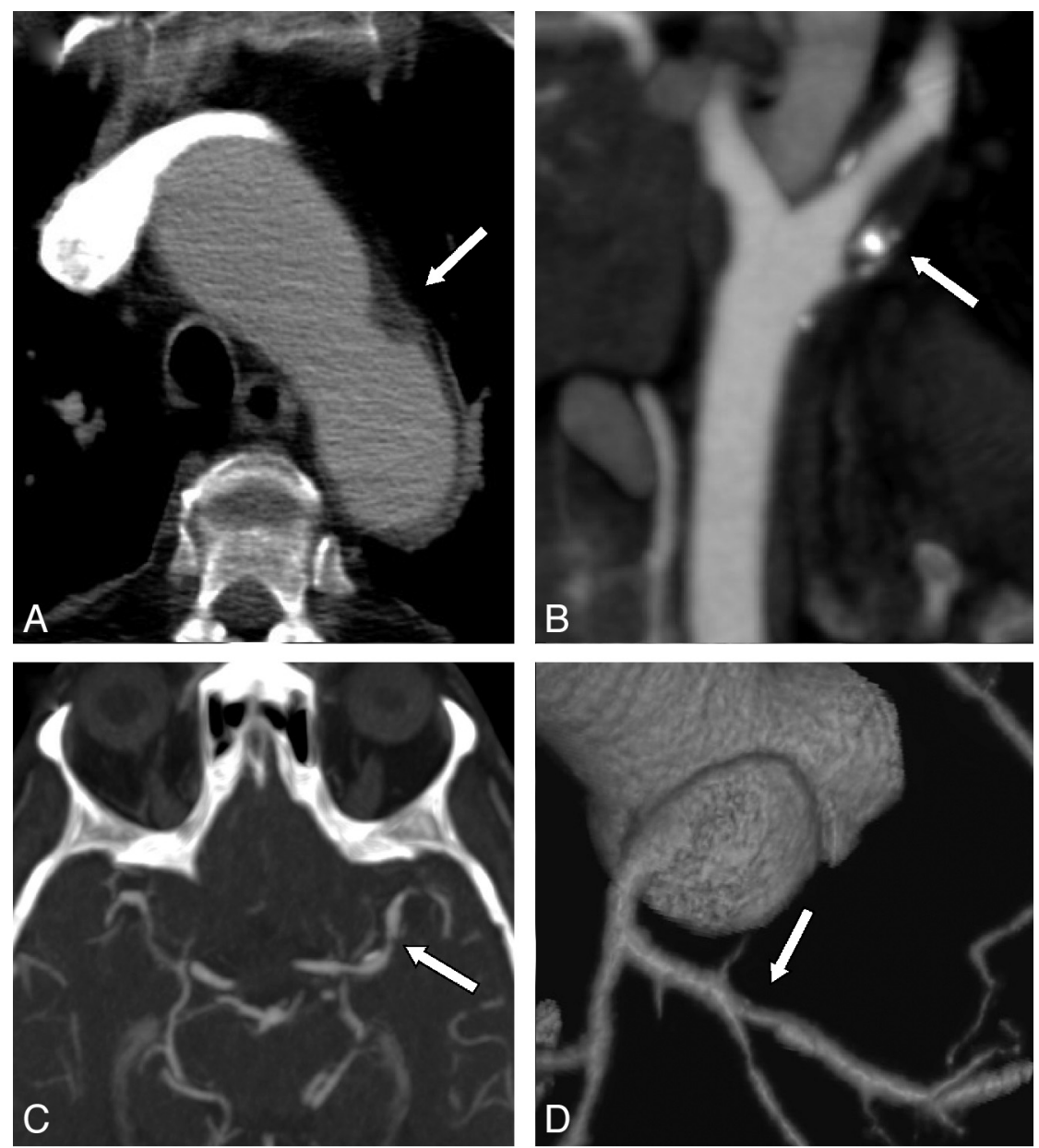

FIG 1. MDCT images (40 sections) show atherosclerosis of the aortic arch $(A)$, internal carotid artery $(B)$, left middle cerebral artery $(C)$, and circumflex coronary artery $(D)$.

The prevalence of $\geq 4 \mathrm{~mm}$ aortic arch atheroma, $\geq 50 \%$ coronary artery stenosis, $\geq 50 \%$ cervical artery stenosis, and $\geq 50 \%$ intracranial artery stenosis was $23.3 \%, 14.1 \%, 23.9 \%$, and $13.6 \%$, respectively (Fig 1).

Forty-four patients $(48.3 \%)$ had no atherosclerotic level. The prevalence of 1 , 2, 3, and 4 atherosclerotic levels was $35.2 \%, 12.1 \% 4.4 \%$, and $0 \%$, respectively. Demographic and clinical data according to the number of atherosclerotic levels are detailed in Table 2. Results did not differ according to diagnosis (TIA vs ischemic stroke) $(P=.75)$. The number of atherosclerotic levels was not associated with classic vascular risk factors besides age. Patients with $\geq 2$ atherosclerotic levels were older than patients with $<2$ atherosclerotic levels $(P=.04)$. After adjustment for main confounding variables, this association was not found.

Among patients with 1 atherosclerotic level, $47.6 \%$ had $\geq 4 \mathrm{~mm}$ aortic arch atheroma, $20.7 \%$ had $\geq 50 \%$ coronary artery stenosis, $26.7 \%$ had $\geq 50 \%$ cervical artery stenosis, and $26.7 \%$ had $\geq 50 \%$ intracranial artery stenosis. Among patients with 2 atherosclerotic levels, $63.6 \%$ had both $\geq 4 \mathrm{~mm}$ aortic arch atheroma and $\geq 50 \%$ cervical artery stenosis. Among patients with 3 atherosclerotic levels, 50\% had both $\geq 4 \mathrm{~mm}$ aortic arch atheroma and $\geq 50 \%$ cervical and coronary artery stenosis. The distribution of artery disease combinations in patients with $\geq 2$ atherosclerotic levels was significantly different $(P=.022)$ (Fig 2). Aortic arch atheroma $\geq 4 \mathrm{~mm}$ and $\geq 50 \%$ cervical artery stenosis were most often associated.

\section{DISCUSSION}

We have shown that $16.5 \%$ of patients with stroke or TIA without evident cardioembolic source have $\geq 2$ atherosclerotic levels by use of MDCT. The combination of $\geq 4$ - $\mathrm{mm}$ aortic arch atheroma and $\geq 50 \%$ cervical stenosis was more often found.

The extent of atherosclerosis is heavier than in a previous study assessing 3 arterial levels (aorta, coronary, and cervical

done or was not interpretable in 5 patients. The characteristics of the remaining 91 patients are shown in Table 1. Mean age was 67.4 ( \pm 11.0$)$ years, 75 patients $(83.3 \%)$ were men, 38 (41.1\%) were diagnosed with ischemic stroke, and 53 (58.9\%) were diagnosed with a TIA. The mean radiation dose to the patients was 18.7 ( \pm 5.0$) \mathrm{mSv}$. arteries) by use of a CTA protocol in patients with suspicion for TIA or stroke. ${ }^{13}$ In this previous study, among 79 patients, 26 (33\%) had 1 atherosclerotic level, mainly a $\geq 50 \%$ coronary artery stenosis. Only 7 patients (9\%) had at least 2 atherosclerotic locations. The enrollment of patients with suspicion of TIA or stroke confirmed in only $60 \%$ of cases and the lack of assess- 
Table 2: Number of atherosclerotic levels and risk factors

\begin{tabular}{lccc} 
& \multicolumn{2}{c}{$\begin{array}{c}\text { Number of Atherosclerotic } \\
\text { Levels }\end{array}$} & \\
\cline { 2 - 3 } & $<2$ & $\geq 2$ & P Value \\
\cline { 2 - 3 } & 76 & 15 & \\
Number of patients & $66.4(10.9)$ & $72.5(10.2)$ & .04 \\
Age, $y$, mean (SD) & & & \\
Sex & $14(18.4)$ & $1(6.4)$ & .45 \\
$\quad$ Female, $n$ (\%) & $61(81.3)$ & $14(93.3)$ & \\
$\quad$ Male, $n$ (\%) & $31(41.3)$ & $7(46.7)$ & .77 \\
Hypertension, $n$ (\%) & $9(12)$ & 0 & .34 \\
Diabetes, $n$ (\%) & $1.22(0.4)$ & $1.23(0.4)$ & .9 \\
LDL cholesterol (g/L), & & & \\
mean (SD) & & & \\
Smoking & $15(20.3)$ & $4(26.7)$ & .75 \\
$\quad$ Former, $n$ (\%) & $17(23.0)$ & $4(26.7)$ & \\
$\quad$ Current, $n$ (\%) & $42(56.8)$ & $7(46.7)$ & \\
$\quad$ Never, $n$ (\%) & $8.4(21.4)$ & $4(3.8)$ & .83 \\
CRP (mg/L), mean (SD) & $20(27.0)$ & $6(40)$ & .07 \\
History of vascular disease, & & & \\
$n$ (\%) & &
\end{tabular}

Note:-CRP indicates C-reactive protein; LDL, low-density lipoprotein; SD, standard deviation.

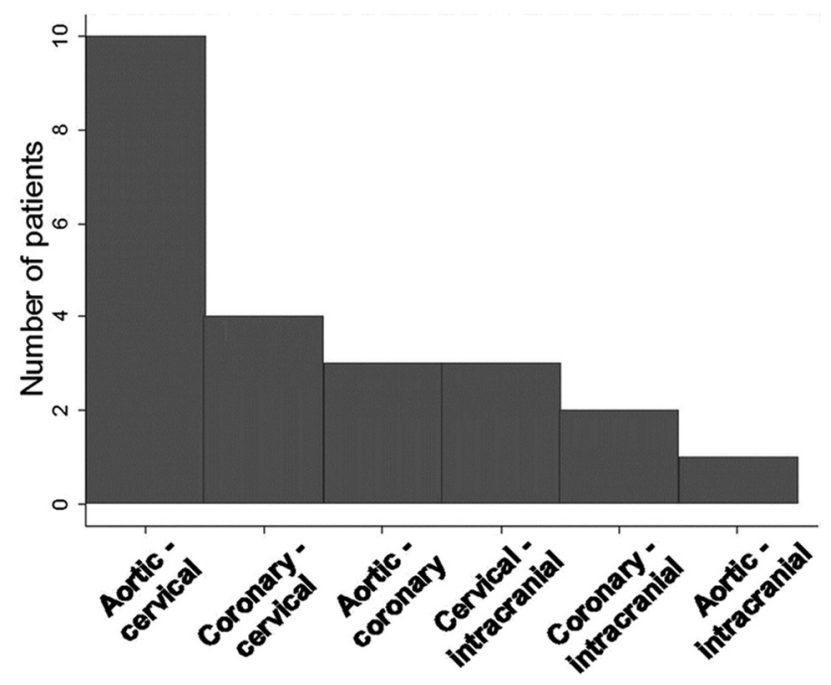

FIG 2. Arterial disease combinations in patients with $\geq 2$ atherosclerotic levels.

ment of the intracranial arteries might account for this difference.

The association between aortic and cervical atherosclerosis was not found by MDCT in this previous study (Adraktas et al) ${ }^{13}$ but has been commonplace in studies using transesophageal echocardiography and carotid ultrasonography in patients with stroke. ${ }^{14-16}$ A prospective study and a case-control study have shown that aortic plaques were more likely detected in patients with stroke with $\geq 50 \%$ carotid artery stenosis compared with $<$ $50 \%$ carotid artery stenosis. ${ }^{14,15}$ Similar results have been shown with mobile thrombi. This association was also found in a third study. ${ }^{16}$

The distribution of atherosclerotic disease is in line with previous studies using MDCT in patients with stroke apart from cervical stenosis. Studies have detected $\geq 50 \%$ intracranial artery stenosis in $10 \%$ of cases $^{17}$ and $\geq 4$-mm aortic arch plaques in approximately $20 \%$ of cases, ${ }^{4,18}$ which could have contributed to stroke occurrence. Asymptomatic coronary artery disease has also been detected in $18 \%-37.5 \%$ of cases. ${ }^{18-22}$ The prevalence of at least $1 \geq 50 \%$ cervical artery stenosis assessed by MDCT in patients with stroke is not available.

The main limitation of our CT protocol was the required radiation dose. Despite an attempt to lower the dose by decreasing the milliampere-second setting (from $300 \mathrm{mAs}$ per section), the retrospective helical mode we used for cardiac examination led to high radiation exposure.

A MDCT protocol allows assessment of not only the aortic, cervical, and intracranial arteries as a usual etiologic work-up of TIA and stroke but also of the coronary arteries. The rate of cardiac mortality is twice as high as cerebrovascular mortality in patients with stroke. ${ }^{23,24}$ Detection of asymptomatic coronary artery stenosis could lead to optimized preventive strategies. Indeed, some anatomic patterns of coronary artery disease such as significant left main stenosis or multivessel disease are strong indications for revascularization. ${ }^{25}$

\section{CONCLUSIONS}

According to the 2 parts of our study, MDCT might be used simultaneously for the etiologic work-up of TIA and ischemic stroke and for assessment of the extent of atherosclerosis. It could help to screen for high-risk patients who could benefit from more aggressive preventive strategies. More research is needed to assess whether the extent of atherosclerosis is associated with classic vascular risk factors and whether it could be an independent prognostic factor regarding vascular outcome in patients with stroke.

Disclosures: Philippe Charles Douek-UNRELATED: Consultancy: Genfit; Grants/ Grants Pending: ANR*; Patents (planned, pending or issued): MR micro antenna (2002) not related to the work; Payment for Development of Educational Presentations: Philips, Comments: workshop on cardiac CT (image processing software in demonstration during workshop); Travel/Accommodations/Meeting Expenses Unrelated to Activities Listed: Philips, Comments: Funding for travel to Radiological Society of North America 2012. *Money paid to institution.

\section{REFERENCES}

1. Nonent M, Serfaty JM, Nighoghossian N, et al. Concordance rate differences of 3 noninvasive imaging techniques to measure carotid stenosis in clinical routine practice: results of the CARMEDAS multicenter study. Stroke 2004;35:682-86

2. Hamon M, Morello R, Riddell JW, et al. Coronary arteries: diagnostic performance of 16- versus 64-section spiral CT compared with invasive coronary angiography-meta-analysis. Radiology 2007;245:720-31

3. Nguyen-Huynh MN, Wintermark M, English J, et al. How accurate is CT angiography in evaluating intracranial atherosclerotic disease? Stroke 2008;39:1184-88

4. Ko Y, Park JH, Yang MH, et al. Significance of aortic atherosclerotic disease in possibly embolic stroke: 64-multidetector row computed tomography study. J Neurol 2010;257:699-705

5. Wardlaw JM, Stevenson MD, Chappell F, et al. Carotid artery imaging for secondary stroke prevention: both imaging modality and rapid access to imaging are important. Stroke 2009;40:3511-17

6. Boussel L, Cakmak S, Wintermark M, et al. Ischemic stroke: etiologic work-up with multidetector CT of heart and extra- and intracranial arteries. Radiology 2011;258:206-12

7. Budoff MJ, Cohen MC, Garcia MJ, et al. ACCF/AHA clinical competence statement on cardiac imaging with computed tomography and magnetic resonance. Circulation 2005;112:598-617

8. Schroeder S, Achenbach S, Bengel F, et al. Cardiac computed tomography: indications, applications, limitations, and training requirements - report of a writing group deployed by the Working 
Group Nuclear Cardiology and Cardiac CT of the European Society of Cardiology and the European Council of Nuclear Cardiology. Eur Heart J 2008;29:531-56

9. Weinreb JC, Larson PA, Woodard PK, et al. American College of Radiology clinical statement on noninvasive cardiac imaging. $\mathrm{Ra}$ diology 2005;235:723-27

10. North American Symptomatic Carotid Endarterectomy Trial (NASCET). Methods, patient characteristics, and progress. Stroke 1991;22:711-20

11. Bartlett ES, Walters TD, Symons SP, et al. Diagnosing carotid stenosis near-occlusion by using CT angiography. AJNR Am J Neuroradiol 2006;27:632-37

12. Fox AJ. How to measure carotid stenosis. Radiology 1993;186: $316-18$

13. Adraktas DD, Brasic N, Furtado AD, et al. Carotid atherosclerosis does not predict coronary, vertebral, or aortic atherosclerosis in patients with acute stroke symptoms. Stroke 2010;41:1604-09

14. Harloff A, Handke M, Geibel A, et al. Do stroke patients with normal carotid arteries require TEE for exclusion of relevant aortic plaques? J Neurol Neurosurg Psychiatry 2005;76:1654-58

15. Demopoulos LA, Tunick PA, Bernstein NE, et al. Protruding atheromas of the aortic arch in symptomatic patients with carotid artery disease. Am Heart J 1995; 129:40 - 44

16. Amarenco P, Cohen A, Tzourio C, et al. Atherosclerotic disease of the aortic arch and the risk of ischemic stroke. $N$ Engl J Med 1994;331:1474-79

17. Homburg PJ, Plas GJ, Rozie S, et al. Prevalence and calcification of intracranial arterial stenotic lesions as assessed with multidetector computed tomography angiography. Stroke 2011;42:1244-50

18. Cho HJ, Lee JH, Kim YJ, et al. Comprehensive evaluation of coro- nary artery disease and aortic atherosclerosis in acute ischemic stroke patients: usefulness based on Framingham risk score and stroke subtype. Cerebrovasc Dis 2011;31:592-600

19. Calvet D, Touzé E, Varenne O, et al. Prevalence of asymptomatic coronary artery disease in ischemic stroke patients: the PRECORIS study. Circulation 2010;121:1623-29

20. Hoshino A, Nakamura T, Enomoto S, et al. Prevalence of coronary artery disease in Japanese patients with cerebral infarction: impact of metabolic syndrome and intracranial large artery atherosclerosis. Circ J 2008;72:404-08

21. Yoon YE, Chang HJ, Cho I, et al. Incidence of subclinical coronary atherosclerosis in patients with suspected embolic stroke using cardiac computed tomography. Int J Cardiovasc Imaging 2011;27: 1035-44

22. Seo WK, Yong HS, Koh SB, et al. Correlation of coronary artery atherosclerosis with atherosclerosis of the intracranial cerebral artery and the extracranial carotid artery. Eur Neurol 2008;59:292-98

23. Dhamoon MS, Sciacca RR, Rundek T, et al. Recurrent stroke and cardiac risks after first ischemic stroke: the Northern Manhattan Study. Neurology 2006;66:641-46

24. Touzé E, Varenne O, Chatellier G, et al. Risk of myocardial infarction and vascular death after transient ischemic attack and ischemic stroke: a systematic review and meta-analysis. Stroke 2005;36:2748-55

25. Wijns W, Kohl P, Danchin N, et al. Guidelines on myocardial revascularization: Task Force on Myocardial Revascularization of the European Society of Cardiology (ESC) and the European Association for Cardio-Thoracic Surgery (EACTS). Eur Heart J 2010;31: 2501-55 